\title{
The correlation between common postoperative complications and quality of life, serum tumor markers, and prognosis in patients with esophageal cancer
}

\author{
Linlin Sun ${ }^{1 \#}$, Fengqin $\mathrm{Xu}^{2 \#}$, Xiajun $\mathrm{Xu}^{3}$, Chunmei Liu ${ }^{2}$ \\ ${ }^{1}$ Department of Oncology, Nanjing Medical University Kangda College First Affiliated Hospital, The First People's Hospital of Lianyungang, \\ Lianyungang, China; ${ }^{2}$ Nursing Department, Nanjing Medical University Kangda College First Affiliated Hospital, The First People's Hospital of \\ Lianyungang, Lianyungang, China; ${ }^{3}$ Department of Thoracic Surgery, The Affiliated Huai'an Hospital of Xuzhou Medical University, Huai'an, \\ China \\ Contributions: (I) Conception and design: L Sun, F Xu; (II) Administrative support: X Xu, C Liu; (III) Provision of study materials or patients: L Sun, \\ F Xu; (IV) Collection and assembly of data: L Sun, F Xu; (V) Data analysis and interpretation: All authors; (VI) Manuscript writing: All authors; (VII) \\ Final approval of manuscript: All authors. \\ "These authors contributed equally to this work. \\ Correspondence to: Xiajun Xu. Department of Thoracic Surgery, The Affiliated Huai'an Hospital of Xuzhou Medical University, No. 62, Huaihai \\ South Road, Huai'an 223002, China. Email: xiajunxu88@163.com; Chunmei Liu. Nursing Department, The First People’s Hospital of Lianyungang, \\ No. 6, Zhenhua East Road, Haizhou District, Lianyungang 222061, China. Email: lygyds@126.com.
}

Background: Esophageal cancer (EC) is highly malignant, with poor prognosis. The main forms of treatment are surgery, radiotherapy, and chemotherapy. In recent years, the incidence and mortality rate of patients with EC has improved. However, the factors that affect the quality of life of EC patients are unclear. This study investigated the postoperative complications and quality of life of EC patients, and identified the related factors. The relationship between complications and quality of life was explored so as to provide guidance for the clinical treatment and rehabilitation these patients.

Methods: A total of 120 patients with EC who underwent surgery at the First People's Hospital of Lianyungang from January 2016 to August 2017 were retrospectively enrolled in this study. The patient's subjective quality of life evaluation was documented and clinical data were collated. The correlation between the incidence of postoperative complications and the patient's quality of life, serum tumor markers, and prognosis was analyzed.

Results: A total of 36 (30\%) EC patients experienced postoperative complications. There were 12 cases of pulmonary complications (10\%), 8 cases of arrhythmia (6.67\%), 3 cases of anastomotic fistula $(2.5 \%)$, 5 cases of pneumothorax and pleural effusion (4.17\%), and 4 cases of gastroesophageal reflux (3.33\%). Incision infection occurred in 2 patients $(1.67 \%)$ and there was 1 case $(0.83 \%)$ of chylothorax. Empyema was reported in 1 patient $(0.83 \%)$. There were significant differences in the overall condition, physical function, fatigue, pain, and swallowing pain between patients with postoperative complications and those without complications $(\mathrm{P}<0.05)$. Preoperative and postoperative expression of the tumor marker CYFRA21-1 was found to be independent risk factors for postoperative complications after EC surgery.

Conclusions: EC is a common malignant tumor with a high incidence of postoperative complications. Patients with high CYFRA21-1 expression should be aware of the higher risk of postoperative complications. Patients with complications have poorer quality of life and obvious symptoms of fatigue, pain, and swallowing pain. Health education and dietary guidance should be provided to such patients to improve their symptoms.

Keywords: Esophageal cancer (EC); complication; quality of life; serum tumor markers; prognosis

Submitted Oct 26, 2021. Accepted for publication Dec 08, 2021.

doi: $10.21037 /$ tcr-21-2562

View this article at: https://dx.doi.org/10.21037/tcr-21-2562 


\section{Introduction}

Esophageal cancer (EC) is one of the most common gastrointestinal malignancies worldwide, and its associated mortality ranks sixth among all malignancies. In 2015, there were 477,900 cases of EC in China and 375,000 patients died of EC in the same year (1). The two common subtypes of EC are esophageal squamous cell carcinoma (ESCC) and esophageal adenocarcinoma (EAC). There is a higher incidence of EAC in the West, while ESCC is more common in China (2). The etiology of EC is complex and can progress from mild atypical hyperplasia to severe atypical hyperplasia, to carcinoma in situ, and finally, to invasive tumor. Early detection, early resection, and multi-modality comprehensive treatments are advocated. However, most patients experience a long asymptomatic period resulting in difficult early diagnosis, and are thus often in the middle to late stages of cancer at diagnosis. The recurrence rate after surgical resection is high and the 5 -year survival rate is about $40 \%$ (3). Postoperative complications mainly include respiratory and cardiovascular complications, anastomotic leakage, gastric emptying disorders, gastroesophageal reflux, recurrent laryngeal nerve injury, diaphragmatic hernia, empyema, and chylothorax. The analysis of perioperative death causes of EC showed that the perioperative mortality of esophageal cancer was $1.94 \%$, about $40 \%$ died of respiratory complications, about $30 \%$ of anastomotic leakage, about $5 \%$ of gastrointestinal bleeding and chylothorax, and about $3 \%$ of circulatory failure and esophageal necrosis (3). These postoperative complications can significantly affect the prognosis and quality of life of these patients. In recent years, with advancements in surgical techniques and instruments, the incidence of total complications has decreased slightly, but the incidence of anastomotic leakage is still high. Therefore, prevention, as well as effective early detection and treatment of postoperative complications is crucial.

At present, assessing the prognosis of EC patients is largely based on routine pathological variables, such as tumor size, grade and tumor stage, as well as the attending doctor's clinical experience (4), which is subject to individual heterogeneity. Therefore, the development of objective indicators is needed in the evaluation and management of patients (5). Tumor markers (TMs) are biomolecules produced by tumor cells during their growth and proliferation, or molecules that are abnormally secreted by the human body due to their reaction with tumor cells. They are expressed at low levels in healthy people, but significantly elevated in cancer patients, thus, indicating the presence of malignancies. Studies have shown that serum TMs play an important role in cancer diagnosis, treatment, prognosis, and monitoring $(6,7)$. Due to the convenience and non-invasive nature of serum TMs, preoperative serum TM levels have been widely used in pancreatic cancer, ovarian cancer, gastrointestinal cancer, breast cancer, and lung cancer. However, there is a paucity of research examining the use of serum TMs in EC. Previous studies have shown that abnormal preoperative levels of serum CA19-9, CA125, SCC, CEA, CA724, CA50 and CYFRA21-1 have certain diagnosis and prognosis value in EC patients $(8,9)$.

This study investigated the relationship between the incidence of common postoperative complications and the quality of life in EC patients. Pre- and postoperative serum TM levels were assessed. These results provide guidance for the clinical treatment and rehabilitation of EC patients. We present the following article in accordance with the STROBE reporting checklist (available at https://tcr. amegroups.com/article/view/10.21037/tcr-21-2562/rc).

\section{Methods}

\section{Research subjects}

A total of 120 EC patients who underwent EC surgery at the First People's Hospital of Lianyungang from January 2016 to August 2017 were retrospectively enrolled. The following inclusion criteria were applied: (I) the lesion does not invade important organs ( $\mathrm{T} 0$ to $\mathrm{T} 4 \mathrm{a}$ ); there are none or few lymph node metastases (N0 to N2); and no metastasis is detected in other organs (M0); (II) patients with recurrence or uncontrolled radiotherapy, without obvious local external or distant metastasis; (III) no serious major organ dysfunction nor significant co-morbidities, and the patient can tolerate the operation; (IV) patients with comprehensive clinical data including gender, age, previous history, preoperative co-morbidities, pulmonary function, surgical records, serum protein, start time of postoperative enteral nutrition, preoperative and postoperative stages, tumor length, pathological type, resection margin, and lymph node metastasis; and (V) clear pathology before and after the operation is available.

The following patients were excluded: (I) patients not diagnosed with EC; (II) patients with benign pathology after the operation; (III) patients with incomplete clinical data; and (IV) patients with surgical contraindications. Contraindications to surgery included the following: (I) poor general physical condition and nutrition, and 
158 cases of postoperative patients with esophageal cancer in oncology department (January 2016 to August 2017)

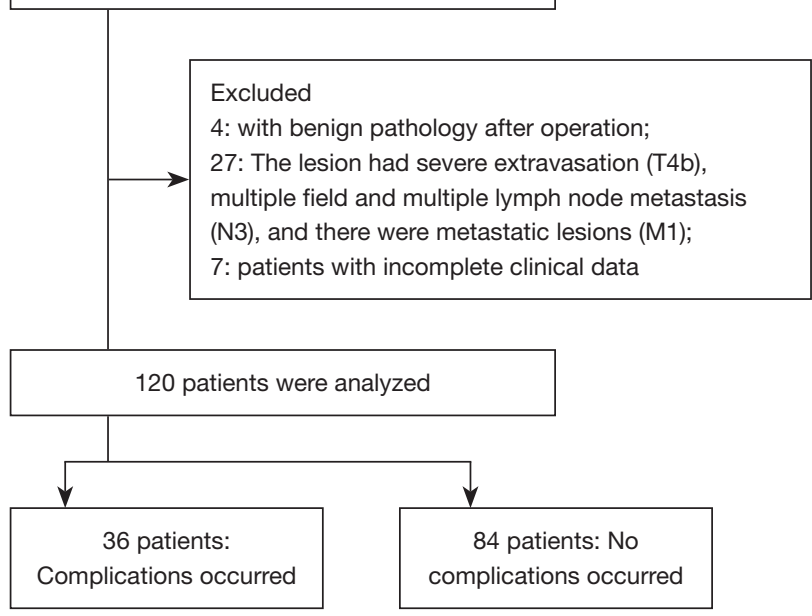

Figure 1 Study flowchart.

presenting with cachexia; (II) the lesion had severe extravasation (T4b), multiple field and multiple lymph node metastasis (N3), and the presences of metastatic lesions (M1); (III) serious dysfunction of important organs, such as severe low pulmonary function, heart failure, myocardial infarction within half a year, severe renal insufficiency, severe liver cirrhosis, etc.; and (IV) other relative contraindications including cervical lymph node metastasis in lower thoracic EC, rich and strong para-arterial lymph node metastasis in cervical EC, perforation of esophageal tumor into the lungs to form an abscess and other infectious foci. See Figure 1 for the patient selection process.

This study was conducted in accordance with the Declaration of Helsinki (as revised in 2013) and was approved by the Ethics Committee of Nanjing Medical University Kangda College First Affiliated Hospital, The First People's Hospital of Lianyungang (2016015). Informed consent was obtained from all patients.

\section{Treatment mode}

All patients were administered general anesthesia prior to complete tumor resection and regional lymph node dissection. There were 45 cases in the three incision oral endoscopy group (right chest and abdominal incision + left neck anastomosis), 65 cases in the three incision open group (right chest and abdominal incision + left neck anastomosis), 7 cases in the right chest upper abdomen median left neck anastomosis + three field dissection, 3 cases in the left chest incision + left neck anastomosis, and 2 cases in the left chest incision + infra arch anastomosis. Left thoracic incision + posterior remnant stomach anastomosis was performed in 1 case. Patients were given standardized and rational use of antibiotics during the perioperative period, postoperative deep vein and duodenal tube feeding nutritional support, and postoperative oxygen mask atomization to assist in sputum excretion. The gastric tube was removed on postoperative day 6 , and patients were offered food on day 7 , gradually transitioning from a liquid diet to a normal diet. Any complications were monitored and treated.

\section{Observation indexes}

\section{Complications}

Patients were monitored for the following postoperative complications.

Anastomotic fistula can cause a large amount of purulent liquid or digestive juices to leak out from the incision or drainage tube. The patient may experience continuous or repeated episodes of pyrexia above $38^{\circ} \mathrm{C}$. Gastroscopy or upper gastrointestinal angiography confirms the presence of a fistula or chest puncture.

Gastroesophageal reflux occurs after eating, where food or gastric juices flow back into the pharynx or mouth, accompanied by a burning sensation or pain behind the sternum and difficulty swallowing.

Arrhythmia is diagnosed by electrocardiography (ECG) or onset of symptoms and discomfort which require treatment.

Pneumothorax and pleural effusion are characterized by spontaneous symptoms, such as shortness of breath and chest tightness that requires treatment.

Incision infection is characterized by a red, swollen, hot, and/or painful incision site. Pus may be visible at the site of surgical incision.

Chylothorax results in shortness of breath and dyspnea. A chylothorax test is performed to confirm a positive diagnosis.

Empyema of the pleural cavity is caused by purulent infection of the pleural cavity.

\section{Quality of life assessment scale}

The quality of life was assessed using the Quality of Life Core Scale (QLQ C30) and the EC specific scale (ose-18) developed by the European Cancer Research and Treatment Organization. The QLQ C30 includes one overall condition 
index, five functional indexes (body, role, emotion, cognition, and society), three general condition indexes (fatigue, nausea and vomiting, and pain), and six single common symptom indexes (dyspnea, insomnia, loss of appetite, constipation, diarrhea, and economic difficulties). In addition to the overall indicators of quality of life ranging from "very poor" (1 point) to "very good" (7 points), each of the above indicators is divided into different options, namely, "not at all" which results in 1 point; "not obvious" resulting in 2 points; "relatively obvious" resulting in 3 points; and "very significant" resulting in 4 points (10). The ose-18 scale has four symptom indicators (swallowing pain, eating difficulty, reflux, and pain) and six single indicators (swallowing difficulty, swallowing asphyxia, dry mouth, taste disorder, cough, and speaking difficulty). Similar to the core scale, each indicator in this scale has four different options. All patients completed the surveys before their operation, and again at 2, 4,12 , and 24 weeks after their operation. The collected data were converted into $0-100$ linear indicators according to the scoring guidelines of the European Organization for Cancer Research and Treatment (11). The higher the functional index and overall condition index after replacement, the better the postoperative quality of life.

\section{Serum tumor markers}

The medical records of the EC patients and the levels of blood TMs were collated, including alpha fetoprotein (AFP), carcinoembryonic antigen (CEA), carbohydrate antigen 19-9 (CA19-9), CA125 (carbohydrate antigen 125), squamous cell carcinoma antigen (SCC), and CYFRA21-1.

\section{Prognosis}

The prognosis of patients was assessed according to the recurrence and survival status. Patients were followed up via outpatient clinics or telephone consultations every 4 months in the first 2 years, and then every 6 months over the next 2 years. Overall survival (OS) was defined as the time interval from the date of surgery to death or the last follow-up. The follow-up period was 36 months or up until August 2020.

\section{Statistical analysis}

After sorting and numbering the questionnaires, statistical analyses were conducted on the data using the Statistical Program for the Social Sciences version 22.0 software (SPSS Inc., Chicago, IL, USA). Measurement data with a normal distribution were described by mean \pm standard deviation $\left(\bar{x}_{ \pm s}\right)$, and categorical data were described as frequencies and percentages. The $t$-test for independent samples was used for comparisons between groups. The chi-square test was used for comparisons of frequency data between groups. The statistically significant indexes of univariate analyses are included, and the multivariate analysis was carried out by binary logistic. A P value $<0.05$ was considered statistically significant.

\section{Results}

\section{Basic patient characteristics}

A total of 120 EC patients were enrolled in this study, including 84 males and 36 females, with an average age of 61.24 years (range, $42-78$ years). There were 22 cases of upper thoracic segment, 57 cases of middle thoracic segment, and 41 cases of lower thoracic segment. All patients were confirmed by pathological examination, and the tumor resection rate was $100 \%$. There were no significant differences in the general characteristics of the patients (Table 1).

\section{Incidence of complications}

Postoperative complications occurred in $36(30 \%)$ patients. There were 12 cases of pulmonary complications (10\%), 8 cases of arrhythmia (6.67\%), 3 cases of anastomotic fistula (2.5\%), 5 cases of pneumothorax and pleural effusion $(4.17 \%)$, and 4 cases of gastroesophageal reflux (3.33\%). Incision infections occurred in 2 patients $(1.67 \%)$. There was 1 case of chylothorax $(0.83 \%)$. Empyema occurred in 1 patient $(0.83 \%)$. All patients were cured and discharged after treatment.

\section{Quality of life score}

There were no significant differences in the preoperative quality of life indexes between patients who had postoperative complications and those who did not. However, postoperatively, there were significant differences in the overall condition, physical function, fatigue, pain, and swallowing pain between the two groups.

The average scores for fatigue, pain, and swallowing pain in the quality of life survey increased rapidly in both groups postoperatively, and was most obvious at about 2 weeks after the operation. Furthermore, the scores were higher in patients in the complication group compared to 
Table 1 Baseline characteristics of the participants with postoperative complications and those without complication $\left(\bar{x}_{ \pm \mathrm{s}}\right)$

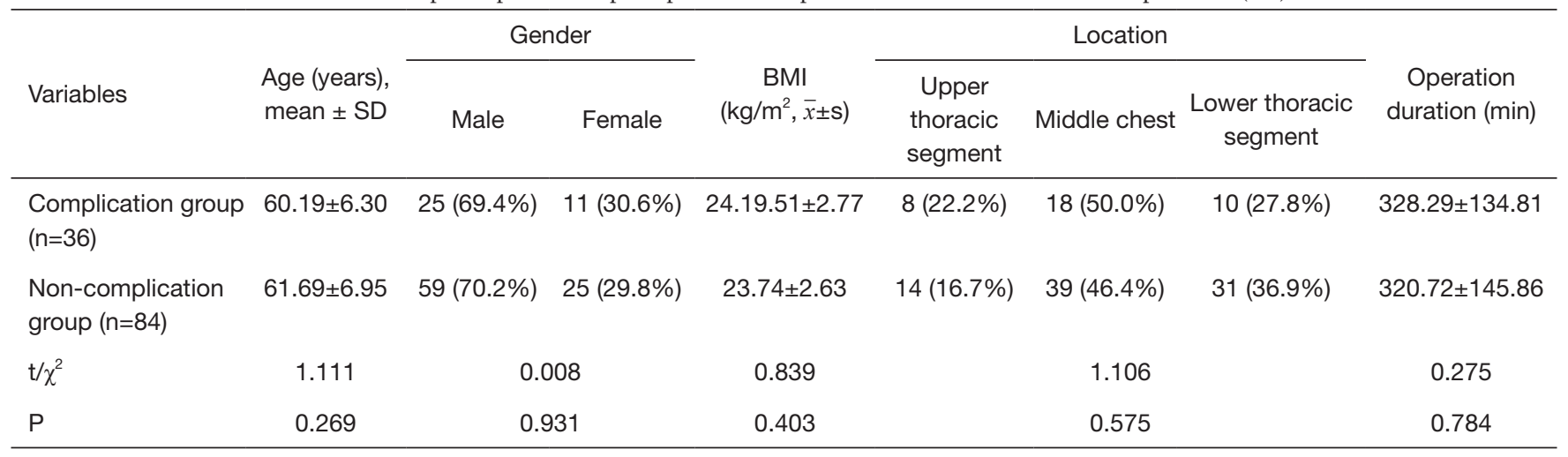

$\mathrm{SD}$, standard deviation; BMI, body mass index.

the non-complication group, especially in the early stages after the operation (0-12 weeks). Thereafter, the data for the two groups became to close, such that by 24 weeks after the operation, the quality of life of patients with complications was similar to that of patients without complications (Table 2).

\section{Analysis of serum tumor markers}

Before operation, the level of serum CYFRA-21 in patients with postoperative complications was significantly higher than that in patients without postoperative complications $(\mathrm{P}<0.05)$.

Postoperatively, the serum levels of CEA, SCC and CYFRA-21 were significantly higher in patients with postoperative complications compared to patients who did not experience postoperative complications $(\mathrm{P}<0.05$; Table 3$)$.

\section{Survival rate}

At the end of follow-up period, $78(65.0 \%)$ of the 120 EC patients survived and $42(35.0 \%)$ died. Complete followup data was available for all patients. The 1-, 2-, and 3 -year overall survival rates for patients with complications were $80.56 \%, 69.44 \%$, and $63.89 \%$, respectively. The $1-$, 2 -, and 3-year overall survival rates for patients without complications were $85.71 \%, 72.62 \%$, and $65.48 \%$, respectively. There was no significant difference between the two groups (Table 4).

\section{Analysis of the risk factors related to the incidence of complications}

Logistic regression analysis showed that CYFRA21-1 levels were an independent risk factor for postoperative complications in EC patients (Table 5).

\section{Discussion}

Outcome indicators such as overall survival and tumorfree survival are widely used in cancer research. However, in diseases such as EC, due to its high incidence of complications and mortality, survival alone cannot effectively describe patient outcomes (12). Therefore, the quality of life is recommended by the U.S. Food and Drug Administration as a secondary index to evaluate cancer data (13). The quality of life refers to a patient's self-interpretation of their satisfaction regarding their needs. Quality of life indicators can provide evaluation criteria for various treatment methods.

During the 24-week follow-up period in this study cohort, the overall condition and physical function of the patients in the complication group was lower than those in the noncomplication group. Fatigue, pain, and swallowing pain were also more intense than that experienced by patients in the non-complication group, and this was most obvious at about 2 weeks post-operation. Thereafter, the data from the two groups became close, such that by 24 weeks post-operation, the complication group had a similar quality of life compared to the non-complication group. After EC surgery, patients can develop symptoms and complications that can affect the quality of life, such as pain. Therefore, while the overall health status score of patients with complications is low, if the complications are controlled, the overall health status of the quality of life will increase.

Previous studies have analyzed the role of TMs in the survival prognosis of patients with lung cancer, gastric cancer, and cervical cancer. In fact, it has been demonstrated 


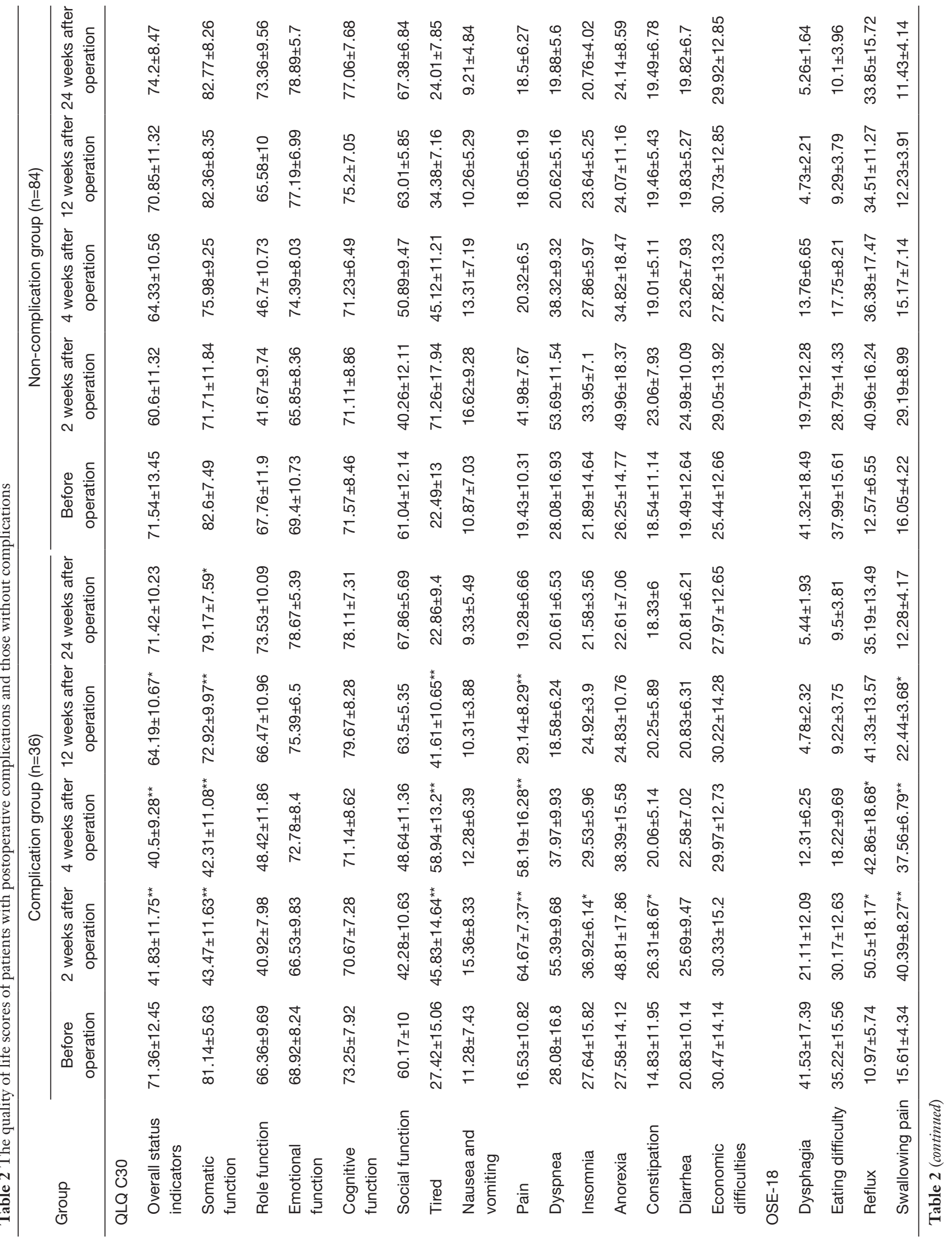




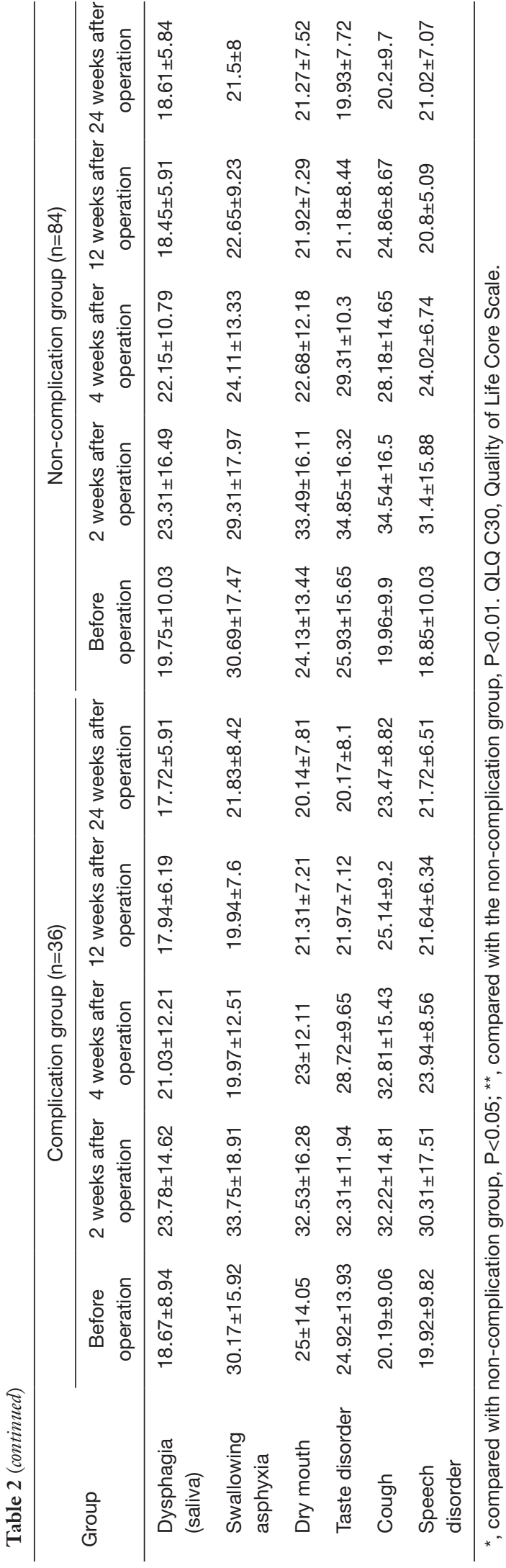

that high CYFRA21-1 serum titer corresponds to poor prognosis of patients with non-small cell lung cancer and colorectal cancer (14). This current study showed that the elevated levels of CYFRA21-1 were correlated to patients who were more likely to have complications. Recently, Vercauteren et al. (15) reported that elevated serum CYFRA21-1 levels were associated with pulmonary fibrosis. Therefore, high serum CYFRA21-1 levels may be associated with tissue disorders at the anastomotic site, such as microvascular agenesis. Future studies examining the biological significance of serum CYFRA21-1 are warranted. For patients with high serum CYFRA21-1 levels, pressurized microvascular anastomosis between the neck and the replacement esophagus should be considered to avoid anastomotic leakage.

In our study cohort, the incidence of postoperative pulmonary complications and arrhythmia was very high, $10 \%$ and $6.67 \%$ respectively. Derogar and colleagues (16) suggested that postoperative complications of EC can negatively affect the long-term survival quality of patients after EC, and Lerut et al. (17) reported a certain correlation between the early recurrence of EC and the incidence of complications. Therefore, reducing and managing these complications is of vital importance. To reduce these surgical complications, in addition to continuously improving surgical procedures, other preoperative interventions can be performed. For example, van Adrichem et al. (18) demonstrated that pulmonary complications after EC can be reduced by preoperative inspiratory muscle training. Jafari and colleagues analyzed the medical records of EC patients in the United States and concluded that there were less complications and lower mortality associated with partial esophagectomy and intrathoracic anastomosis compared to subtotal esophagectomy and neck anastomosis (19). Despite improvements in long-term survival in surgically treated EC patients (20), the high incidence of associated complications (21) highlights the importance of postoperative quality of life management. The results of this study emphasized the need to take preventive measures to control postoperative complications in order to improve the quality of life of EC survivors. Customized follow-up consultations, as well as physical and mental rehabilitation should be provided for patients with persistent complications.

\section{Conclusions}

EC is a common malignant tumor and the high incidence 
Table 3 Serum tumor markers in patients with postoperative complications and those without complications

\begin{tabular}{|c|c|c|c|c|c|c|}
\hline Time & Groups & CEA $(\mu g / L)$ & CA19-9 (U/mL) & CA125 (U/mL) & $\operatorname{SCC}(\mu \mathrm{g} / \mathrm{L})$ & CYFRA21-1 $(\mu \mathrm{g} / \mathrm{L})$ \\
\hline \multirow{2}{*}{ Before operation } & $\begin{array}{l}\text { Non-complication } \\
\text { group }(n=84)\end{array}$ & $3.5 \pm 1.06$ & $86.52 \pm 7.25$ & $78.32 \pm 6.2$ & $1.49 \pm 0.59$ & $2.16 \pm 1.07$ \\
\hline & $\mathrm{t}$ & 0.597 & 0.180 & 0.300 & 1.330 & 14.709 \\
\hline \multirow[t]{2}{*}{$\begin{array}{l}\text { Three days after } \\
\text { operation }\end{array}$} & $\begin{array}{l}\text { Complication group } \\
\qquad(n=36)\end{array}$ & $2.56 \pm 0.61$ & $41.17 \pm 3.78$ & $31.03 \pm 4.14$ & $1.34 \pm 0.38$ & $4.12 \pm 0.74$ \\
\hline & $\begin{array}{l}\text { Non-complication } \\
\text { group }(n=84)\end{array}$ & $1.28 \pm 0.34$ & $41.51 \pm 4.88$ & $31.46 \pm 4.58$ & $1.1 \pm 0.36$ & $2.96 \pm 0.41$ \\
\hline
\end{tabular}

CEA, carcinoembryonic antigen; CA19-9, carbohydrate antigen 19-9; CA125, carbohydrate antigen 125; SCC, squamous cell carcinoma antigen.

Table 4 Clinical outcomes of patients with postoperative complications and those without complications

\begin{tabular}{lccc}
\hline Groups & 1-year survival rate $(\%)$ & 2-year survival rate (\%) & 3-year survival rate (\%) \\
\hline Complication group $(\mathrm{n}=36)$ & $29(80.56)$ & $25(69.44)$ & $23(63.89)$ \\
Non-complication group $(\mathrm{n}=84)$ & $72(85.71)$ & $61(72.62)$ & $55(65.48)$ \\
$\chi^{2}$ & 0.503 & 0.125 & 0.028 \\
$\mathrm{P}$ & 0.478 & 0.724 & 0.867 \\
\hline
\end{tabular}

Table 5 The correlation between serum tumor markers and the incidence of complications

\begin{tabular}{lccccc}
\hline Related factor & $\beta$ & SE & Ward & OR & $95 \% \mathrm{Cl}$ \\
\hline Preoperative CYFRA21-1 & 4.131 & 0.705 & 5.329 & 6.224 & $1.562-24.786$ \\
Postoperative CEA & 2.048 & 0.360 & 1.269 & 7.754 & $0.539-11.442$ \\
Postoperative SCC & 1.651 & 0.575 & 3.031 & 2.720 & $0.882-8.388$ \\
Postoperative CYFRA21-1 & 3.130 & 0.637 & 24.181 & 3.044 & $0.913-10.152$ \\
\hline
\end{tabular}

CEA, carcinoembryonic antigen; SCC, squamous cell carcinoma antigen; SE, standard error; OR, odds ratio; Cl, confidence interval.

of postoperative complications, including symptoms of fatigue, pain, and swallowing pain, can cause significant strain on the patient's quality of life. Patients with high serum CYFRA21-1 levels should be aware of potential postoperative complications. Health education and dietary guidance should be provided to improve the patient's symptoms.

There were some imitations in this study. This investigation was a small, retrospective, single-centered study, which may have been affected by the experience of the doctors, available medical records, and other confounding factors. Large, multi-centered studies are needed in the future to verify the significance of these results in clinical diagnosis and treatment. This study identified the factors related to the incidence of postoperative complications after EC surgery. Future work will involve confirmation of these results using larger samples, as well as developing and verifying a mathematical model to predict the risk of 
postoperative complications in EC patients.

\section{Acknowledgments}

Funding: None.

\section{Footnote}

Reporting Checklist: The authors have completed the STROBE reporting checklist. Available at https://tcr. amegroups.com/article/view/10.21037/tcr-21-2562/rc

Data Sharing Statement: Available at https://tcr.amegroups. com/article/view/10.21037/tcr-21-2562/dss

Conflicts of Interest: All authors have completed the ICMJE uniform disclosure form (available at https://tcr.amegroups. com/article/view/10.21037/tcr-21-2562/coif). The authors have no conflicts of interest to declare.

Ethical Statement: The authors are accountable for all aspects of the work in ensuring that questions related to the accuracy or integrity of any part of the work are appropriately investigated and resolved. The study was conducted in accordance with the Declaration of Helsinki (as revised in 2013). The study was approved by the Ethics Committee of Nanjing Medical University Kangda College First Affiliated Hospital, The First People's Hospital of Lianyungang (2016015). Informed consent was obtained from all patients.

Open Access Statement: This is an Open Access article distributed in accordance with the Creative Commons Attribution-NonCommercial-NoDerivs 4.0 International License (CC BY-NC-ND 4.0), which permits the noncommercial replication and distribution of the article with the strict proviso that no changes or edits are made and the original work is properly cited (including links to both the formal publication through the relevant DOI and the license). See: https://creativecommons.org/licenses/by-nc-nd/4.0/.

\section{References}

1. Chen W, Zheng R, Baade PD, et al. Cancer statistics in China, 2015. CA Cancer J Clin 2016;66:115-32.

2. Ohashi S, Miyamoto S, Kikuchi O, et al. Recent Advances From Basic and Clinical Studies of Esophageal Squamous Cell Carcinoma. Gastroenterology 2015;149:1700-15.
3. Hou H, Meng Z, Zhao X, et al. Survival of Esophageal Cancer in China: A Pooled Analysis on Hospital-Based Studies From 2000 to 2018. Front Oncol 2019;9:548.

4. Ashida A, Boku N, Aoyagi K, et al. Expression profiling of esophageal squamous cell carcinoma patients treated with definitive chemoradiotherapy: clinical implications. Int J Oncol 2006;28:1345-52.

5. Lin DC, Du XL, Wang MR. Protein alterations in ESCC and clinical implications: a review. Dis Esophagus 2009;22:9-20.

6. Ni XG, Bai XF, Mao YL, et al. The clinical value of serum CEA, CA19-9, and CA242 in the diagnosis and prognosis of pancreatic cancer. Eur J Surg Oncol 2005;31:164-9.

7. Nakai $\mathrm{Y}$, Isayama $\mathrm{H}$, Sasaki T, et al. A retrospective analysis of early CA19-9 change in salvage chemotherapy for refractory pancreatic cancer. Cancer Chemother Pharmacol 2013;72:1291-7.

8. Cao X, Zhang L, Feng GR, et al. Preoperative Cyfra21-1 and SCC-Ag serum titers predict survival in patients with stage II esophageal squamous cell carcinoma. J Transl Med 2012;10:197.

9. Thomson IG, Smithers BM, Gotley DC, et al. Thoracoscopic-assisted esophagectomy for esophageal cancer: analysis of patterns and prognostic factors for recurrence. Ann Surg 2010;252:281-91.

10. Aaronson NK, Ahmedzai S, Bergman B, et al. The European Organization for Research and Treatment of Cancer QLQ-C30: a quality-of-life instrument for use in international clinical trials in oncology. J Natl Cancer Inst 1993;85:365-76.

11. Osoba D, Rodrigues G, Myles J, et al. Interpreting the significance of changes in health-related quality-of-life scores. J Clin Oncol 1998;16:139-44.

12. Koller M, Lorenz W. Survival of the quality of life concept. Br J Surg 2003;90:1175-7.

13. Langenhoff BS, Krabbe PF, Wobbes T, et al. Quality of life as an outcome measure in surgical oncology. Br J Surg 2001;88:643-52.

14. Chang CC, Yang SH, Chien CC, et al. Clinical meaning of age-related expression of fecal cytokeratin 19 in colorectal malignancy. BMC Cancer 2009;9:376.

15. Vercauteren IM, Verleden SE, McDonough JE, et al. CYFRA 21.1 in bronchoalveolar lavage of idiopathic pulmonary fibrosis patients. Exp Lung Res 2015;41:459-65.

16. Derogar M, Orsini N, Sadr-Azodi O, et al. Influence of major postoperative complications on health-related quality of life among long-term survivors of esophageal 
cancer surgery. J Clin Oncol 2012;30:1615-9.

17. Lerut T, Moons J, Coosemans W, et al. Postoperative complications after transthoracic esophagectomy for cancer of the esophagus and gastroesophageal junction are correlated with early cancer recurrence: role of systematic grading of complications using the modified Clavien classification. Ann Surg 2009;250:798-807.

18. van Adrichem EJ, Meulenbroek RL, Plukker JT, et al. Comparison of two preoperative inspiratory muscle training programs to prevent pulmonary complications in patients undergoing esophagectomy: a randomized controlled pilot study. Ann Surg Oncol 2014;21:2353-60.

19. Jafari MD, Halabi WJ, Smith BR, et al. A decade analysis of trends and outcomes of partial versus

Cite this article as: Sun L, Xu F, Xu X, Liu C. The correlation between common postoperative complications and quality of life, serum tumor markers, and prognosis in patients with esophageal cancer. Transl Cancer Res 2022;11(1):217-226. doi: $10.21037 /$ tcr-21-2562 total esophagectomy in the United States. Ann Surg 2013;258:450-8.

20. Schmidt HM, Gisbertz SS, Moons J, et al. Defining Benchmarks for Transthoracic Esophagectomy: A Multicenter Analysis of Total Minimally Invasive Esophagectomy in Low Risk Patients. Ann Surg 2017;266:814-21.

21. Ruhstaller T, Widmer L, Schuller JC, et al. Multicenter phase II trial of preoperative induction chemotherapy followed by chemoradiation with docetaxel and cisplatin for locally advanced esophageal carcinoma (SAKK 75/02). Ann Oncol 2009;20:1522-8.

(English Language Editor: J. Teoh) 